\title{
3D Printed Model of Airway for Clinical Simulation
}

\author{
Maheswaran Viyannan ${ }^{\oplus 1}$, Pananghat A. Kumar ${ }^{\oplus 2}$, Sreedharkumar Eswarswamy ${ }^{\circledR 3}$, Gunaseelan Murugesan ${ }^{\odot 4}$, Karthikeyan Ramaraju ${ }^{\circ 5}$ \\ ${ }^{1}$ Assistant Professor, Department of Radiology, PSG Institute of Medical Sciences \& Research, Coimbatore, Tamil Nadu, India, ${ }^{2}$ Advisor, Clinical Simulation Laboratory, \\ PSG Institute of Medical Sciences \& Research, Coimbatore, Tamil Nadu, India, ${ }^{3}$ Senior Engineer, TIFAC Core, PSG College of Technology, Coimbatore, Tamil Nadu, India, \\ ${ }^{4}$ Senior Project Engineer, TIFAC Core, PSG College of Technology, Coimbatore, Tamil Nadu, India, ${ }^{5}$ Professor, Department of Respiratory Medicine, PSG Institute of \\ Medical Sciences \& Research, Coimbatore, Tamil Nadu, India.
}

\section{Abstract}

Background: The present medical curriculum aims at training the students to be proficient in performing techniques required for clinical practice. This is best achieved through clinical simulation, which has emerged as a successful method for clinical learning. Residents in respiratory medicine need to be trained in the procedure of bronchoscopy for which a functional model of the airway is required. Airway mannequins for this purpose can be produced using 3D printing technology, which involves the usage of sophisticated software. Subjects and Methods: Serial axial CT images of the chest, revealing details of the respiratory tract were selected as the base resource to recreate the bronchial tree by $3 \mathrm{D}$ printing. This DICOM (Digital Imaging and Communications in Medicine) images after conversion into STL (Stero lithography) format were transferred into a 3D printer and physical models were made from these data, using Vero clear and rubber. This model which had a life-like form and consistency required for practicing the skill was connected to an airway mannequin using an adaptor to practice the skill. Conclusions: Axial CT scan images provide the base data for reconstructing the airway of a patient, using 3D printing technology and appropriate software. Such reconstructions can be used to produce a functional model of the airway, which can be used for training in bronchoscopy. The training system could be connected to a monitor thereby facilitating tracking of the probe of the bronchoscope. Repeated trials make the trainees perfect their technique. Our attempt to replicating the tracheobronchial tree for such training has been a success.

Keywords: 3D printing, axial CT, Bronchoscopy

Corresponding Author: Pananghat A. Kumar, Advisor, Clinical Simulation Laboratory, PSG Institute of Medical Sciences \& Research, Coimbatore, Tamil Nadu, India.

E-mail: drpakumar@yahoo.com

Received: 02 September 2020

Revised: 13 October 2020

\section{Introduction}

Medical educationists have been stressing the importance and relevance of skills training for students at all levels of their career and the recent document from Medical Council of India (MCI) clearly lists out the various skills which have been mandated for inclusion at different stages of their undergraduate training. ${ }^{[1]}$ Clinical skills are best learned through simulation, which has now emerged as a successful method for practical learning. A major factor in achieving the goals of successful clinical simulation is the scientific accuracy of the equipment like mannequins which are used. Clinical simulation makes use of low fidelity and high fidelity mannequins, which are commercially available. Since quite often, the commercially available mannequins do not fulfill the requirements of the learner, it becomes necessary to innovate simulation mannequins and training models to suit the individual requirements the best.
Among the various methods for innovating mannequins for simulation, 3D printing has come out as a dependable source for generating these valuable resource materials. 3D printing, which has subserved many functions in various industries, is one such technology that has also found its profound impact in the field of medicine as well. This technology, also known as additive manufacturing, first developed in the 1980s has revolutionised the realm of medicine as well. BioMedical Modelling using CT (Computerised tomography) or MRI (Magnetic Resonance Imaging) scan data can produce both physical solid models (Bio models) and CAD (Computeraided design)-compatible virtual models (Bio CAD) that are accurate three-dimensional representations of patient-specific anatomy. Digitalized CT and MRI images have tremendously influenced the diagnostic realm and the practice of medicine. Imaging has made it possible to diagnose accurately and to intervene in a host of pathologies, early enough and efficiently to improve the quality of treatment modalities. 
These reconstructed images could be either virtual or digital in nature and could become the source data for 3D printing. This becomes more relevant, since $3 \mathrm{D}$ printed material offers anatomical accuracy far better than any other known learning medium like plastinated material, with a lot more added advantages. ${ }^{[2-4]}$

The success of simulated learning depends upon how real the training system is, which is based on the quality of the mannequins and the environment which needs to mimic reality. There have been commercially available mannequins and systems that provide an excellent opportunity for selected skills training. These include the basic low fidelity and high fidelity mannequins which function electronically. Mannequins are available for practicing life-saving procedures like cardiopulmonary resuscitation, tracheal intubation, urinary catheterisation, and phlebotomy. Cardiac rhythm generators are also in the use. High fidelity mannequins, which are advanced computerised patient simulators, provide a platform for simulating real-life scenarios in sick patients. While adopting simulationbased teaching in clinical sciences, it becomes necessary to develop learning systems strictly in a need-based manner. This, in fact, becomes one of the challenging responsibilities of the simulation centers.

Our clinical simulation laboratory caters to the skills training requirements for all the years of medical undergraduate students, the residents and the faculty. The laboratory provides training in various courses affiliated with the American Heart Association, as well. Standard airway mannequins, BLS (Basic Life Support) and ACLS (Advanced Cardiovascular Life Support) mannequins have been successfully used for routine skills training. One of the much-required skills in the practice of Respiratory Medicine is to examine the airway to the bronchopulmonary segments (BPS) using a bronchoscope. Bronchoscopy, a vital and important clinical procedure, which finds its utility in various critical specialties like anaesthesiology, respiratory medicine, critical care and thoracic surgery, is one of the popular diagnostic procedures involving endoscopic exploration of the airways and demands working knowledge of the morphological configuration of tracheobronchial segments, along with the expertise in endoscopic maneuvers. Challenges and futuristic directions in bronchoscopic training have been well documented. ${ }^{[5,6]}$ By virtue of its significance, it is important to realise that acquisition of bronchoscopy skills need to start in a simulated setting, instead of training on patients straight away. Anatomical accuracy, fidelity in simulation and cost-effectiveness are the factors that determine the qualities of choice of training modules.

Commercially available simulation models have several demerits like inaccuracy in anatomy of the artificial tracheobronchial tree and exuberant cost. Availability is yet another problem. Attempts were made to produce low-cost 3D-printed models for bronchoscopy training in Canada and in Australian

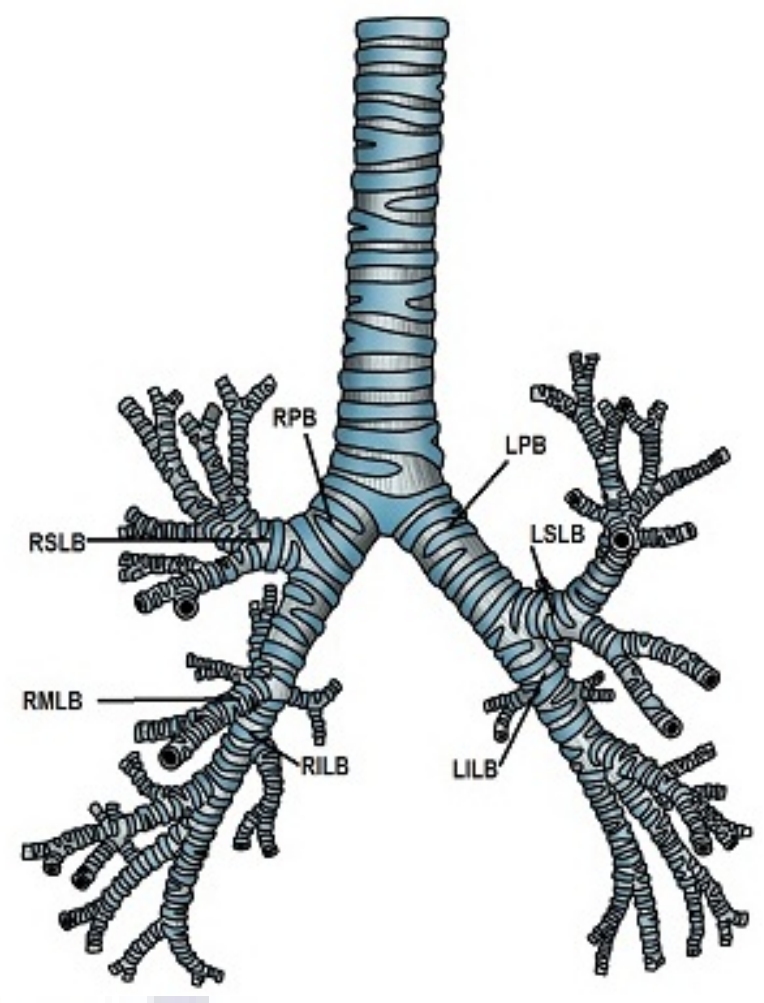

Figure 1: Bronchopulmonary segments.

with varying degrees of success. ${ }^{[7]} \mathrm{We}$ attempted to engineer a mannequin of a tracheobronchial tree that is cost-effective and feasible for mass production. For this purpose, efforts were taken up to create a physical model of the airway up to the subsegmental level, from the CT data of a patient, who had no demonstrable pathologies in the respiratory tract. These would be synthetic body parts reflecting the living anatomy in as lifelike a manner as possible.

Bronchopulmonary segments: Anatomy of the upper airways starting from the beginning of the trachea through the main bronchi till the level of tertiary bronchi describes complex anatomy. ${ }^{[6,7]}$ The segmental bronchi are ten in number on the right and eight on the left [Figure 1]. Generations of these bronchial divisions vary in their length, width and their takeoff angle, thus exhibiting complex anatomical features. [Figure 1]

RPB - Right Principal Bronchus; RSLB - Right Superior Lobar Bronchus; RMLB - Right Middle Lobar Bronchus; RILB - Right Inferior Lobar Bronchus; LPB - Left Principal Bronchus; LSLB -Left Superior Lobar Bronchus; LILB - Left Inferior Lobar Bronchus

The process of bronchoscopy involves the introduction of a fibrotic tube, about the diameter of $6 \mathrm{~mm}$ which houses the 


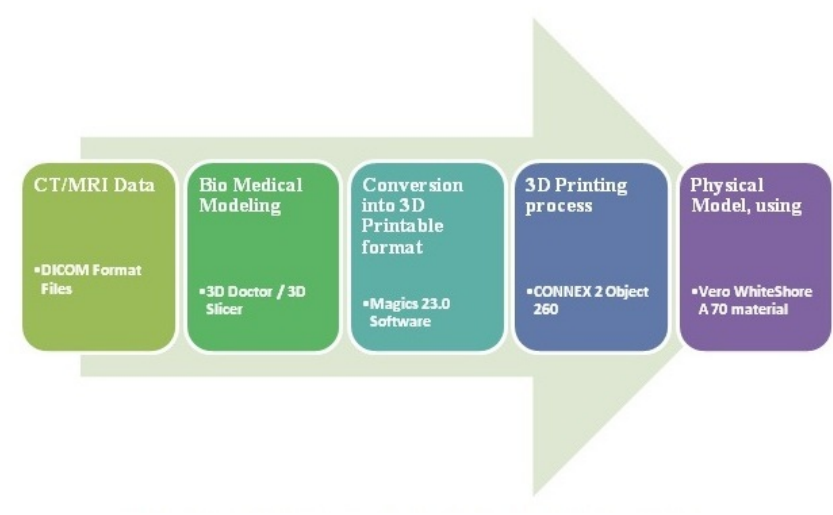

Figure 2: Flow chart showing the steps involved in the 3D printing process

camera at the end of the tube. The manoeuvre of bronchoscopy calls for expertise and a high degree of precision to navigate through the airways, causing the least discomfort to the patient and nil damage to the organs and tissues around the airway lumen. The clinician needs to perfect this skill to be able to succeed in this very important diagnostic procedure. ${ }^{[8,9]}$ For obvious reasons, this skill needs to be perfected on a simulator, specially made for this purpose, before trying it on a patient. ${ }^{[10]}$ The simulator needs to mimic the real appearance and feel of the patient's airway. For this to happen, of all the available methods, 3D printed airway seems to be the best option. Since there is a paucity of such information from our country, we felt it worthwhile to report our experience of having produced a successful working model of a 3D printed airway which was fitted to an airway mannequin to simulate bronchoscopy procedures in our hospital.

\section{Subjects and Methods}

The basic principle of 3D printing is the conversion of digital information to a physical object with the help of computer software. In this regard, radiological images of CT and MRI data in digital imaging and communication in medicine (DICOM) format are an ideal source for 3D printing. DICOM images, which are in digital format, can be easily segmented, edited and converted into 3D printable formats. The process of creating 3D models from digital data involves four major steps: image acquisition, image segmentation, creation of a digital 3D model, and transfer of model data to a 3D printer, which prints out a physical model [Figure 2].

\section{Selection of the CT Images:}

A set of serial axial CT images of the chest, revealing details of the respiratory tract were selected as the base resource to recreate the bronchial tree by $3 \mathrm{D}$ printing. The images were obtained in the suspended inspiration of the respiratory phase which makes the visibility of airways better. We used a window setting which brings out the lung and airways and relatively suppresses the other soft tissues with different CT density. While selecting the set of images, care was taken to avoid artefacts and to see that the images truly represent the morphology of live structures.

\section{Image acquisition:}

The volumetric data of the selected stack of computed tomography (CT) were analyzed to ensure that there are no gaps in anatomical details during acquisition. The essential element in getting a high-resolution $3 \mathrm{D}$ print is thin submillimeter axial sections which are very easy to obtain with modern-day multi-row multi-detector spiral scanners which can produce thousands of such thin section images in a matter of few seconds. The sheer speed of acquisition of these isotropic images virtually eliminates movement-related artefacts like gross body movement or respiratory movement. The medical images coming from the CT scanner consist of grayscale information. These DICOM data were converted into Stereolithography (STL) format, by a process called segmentation, using specialized software, 3D Doctor, which permits the user to create models based on their grey values (Hounsfield units in CT images).

\section{Segmentation - Conversion of image data in DICOM format to STL format:}

The specific information of the region of interest (ROI) from this STL data is refined by a process of Segmentation. Acquired STL data were sent through a series of processing techniques to ensure printability of a 3D model, using the software, 3D Doctor, which is advanced 3D image rendering, processing and analysis software, developed by Able Software Corp., a software development company. This software processed these images in the XY plane (axial images) and added the XZ (coronal) and YZ (sagittal) dimensions to these, thus converting the $2 \mathrm{D}$ image into a $3 \mathrm{D}$ one. This file format is ideal for anatomical geometry because of its simple file structure and flexibility to match any desired contour. The STL format allows describing the most complex geometries accurately. This is necessary since anatomical data is in general very intricate. Accurate segmentation is important in order to extract meaningful information from images. The accuracy in a 3D Doctor model matches the accuracy of an object captured within the scan.

\section{Transfer to the 3D printer:}

The completed STL file was transferred to a 3D printer for production. The last two steps involve the utilization of computer-aided design or computer-aided manufacturing software package.

\section{D printing:}




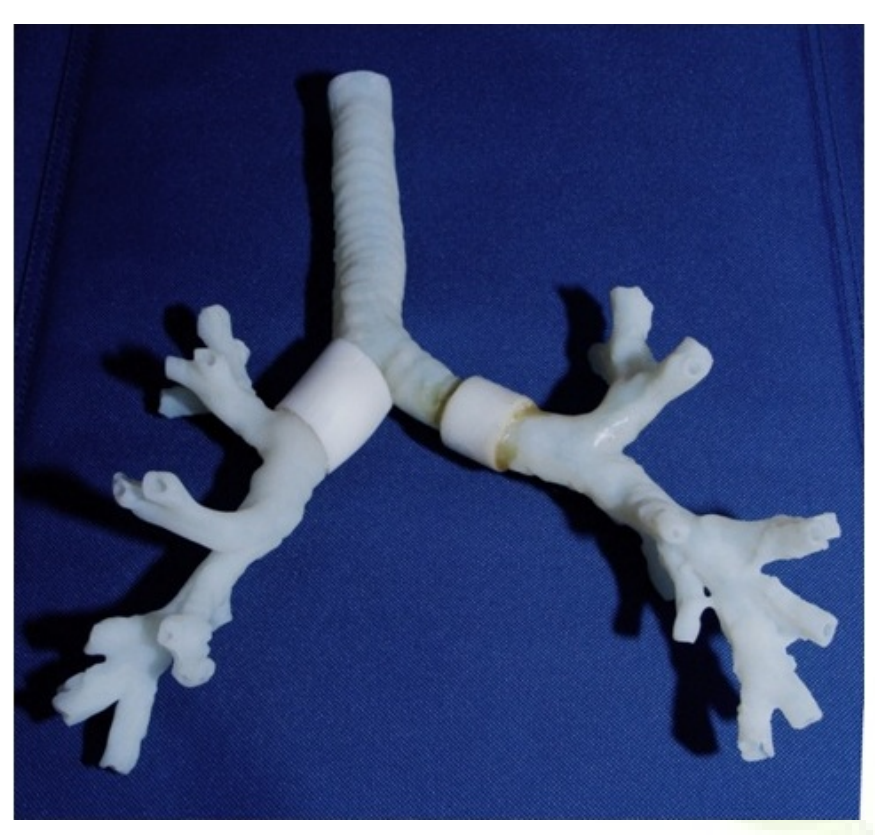

Figure 3: 3D model of the airway.

Prototypes were printed in Acura 60, a material akin to polycarbonate, using the machine ViperSi2. After checking the anatomical accuracy and user-friendliness of the model, thus produced, the final version was made out of a combination of Veroclear and rubber, using Objet 260 Connex 2, a machine from M/s. Stratasys Conex machine. This machine has the widest range of material properties for its class, from rigid to flexible, transparent to opaque, neutral to vibrant, standard to bio-compatible and which can withstand high temperatures was used for further processing. Considering the need for flexibility, Tango black and Vero white plus materials were used in 3D printing of tracheobronchial tree. It is a combination of Polymethyl methacrylate (PMMA) and siliconized rubber. The shore hardness preferred for this process was 70A.

The printing process took around twelve hours and the final product mimics the live airway [Figure 3].

Post-production clearing and cleaning: The output from the machine has been taken for proper cleaning with a water jet to remove the supports and was used for trials of bronchoscopy training.

\section{Assembly of the final product onto the mannequin :}

An airway mannequin, which is used for training endotracheal intubation was chosen to be used for this training. A silicone adapter was made to link the 3D printed model comfortably to this airway mannequin.

\section{Bronchoscopy training:}

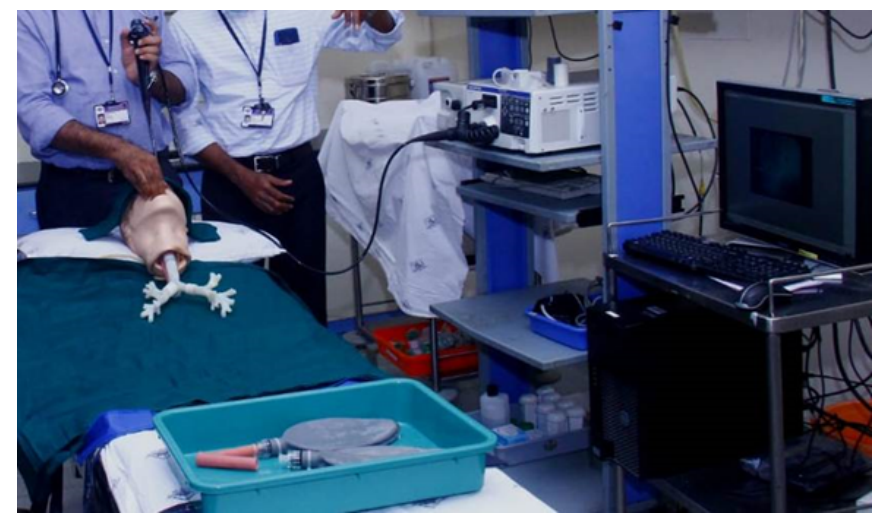

Figure 4: Airway model used for practicing bronchoscopy.

Firstly, a trained physician, using a video bronchoscope, explored the accuracy of the anatomy of the tracheobronchial tree and also the ease of negotiating the scope up to all segmental bronchi on both sides, using an adult video bronchoscope [Figure 4].

Then the trainer simulated a clinical scenario and instructed the learners to reach a particular segment of the lung in the model. For example, they were instructed to reach the lateral segment of the right middle lobe; or the apicoposterior segment of the left lung. The learner used the bronchoscope with precision and their success was gauged by checking on the location of the bronchoscope physically and on the monitor, connected to the bronchoscope. The learners, who were residents in Respiratory medicine were permitted to practice and reach all the segments by rotation [Figure 5].

\section{Results}

A working model of the tracheobronchial tree was constructed using the $3 \mathrm{D}$ printing technology, using material that permitted the bronchoscope to pass through the airway till it reached specific bronchopulmonary segments. Trials of bronchoscopy training using the model proved to be a success taking into consideration the feedback from the interns and the faculty who used the system.

\section{Discussions}

The advent of simulated learning practices in the realm of medical education has revolutionised the training methodologies in clinical medicine since it is now generally acknowledged that the awareness of patient safety largely depends upon the skill of the personnel involved in healthcare delivery. Skills need to be taught using tools that simulate conditions that are true to life so that the training becomes relevant to patient care. Such 

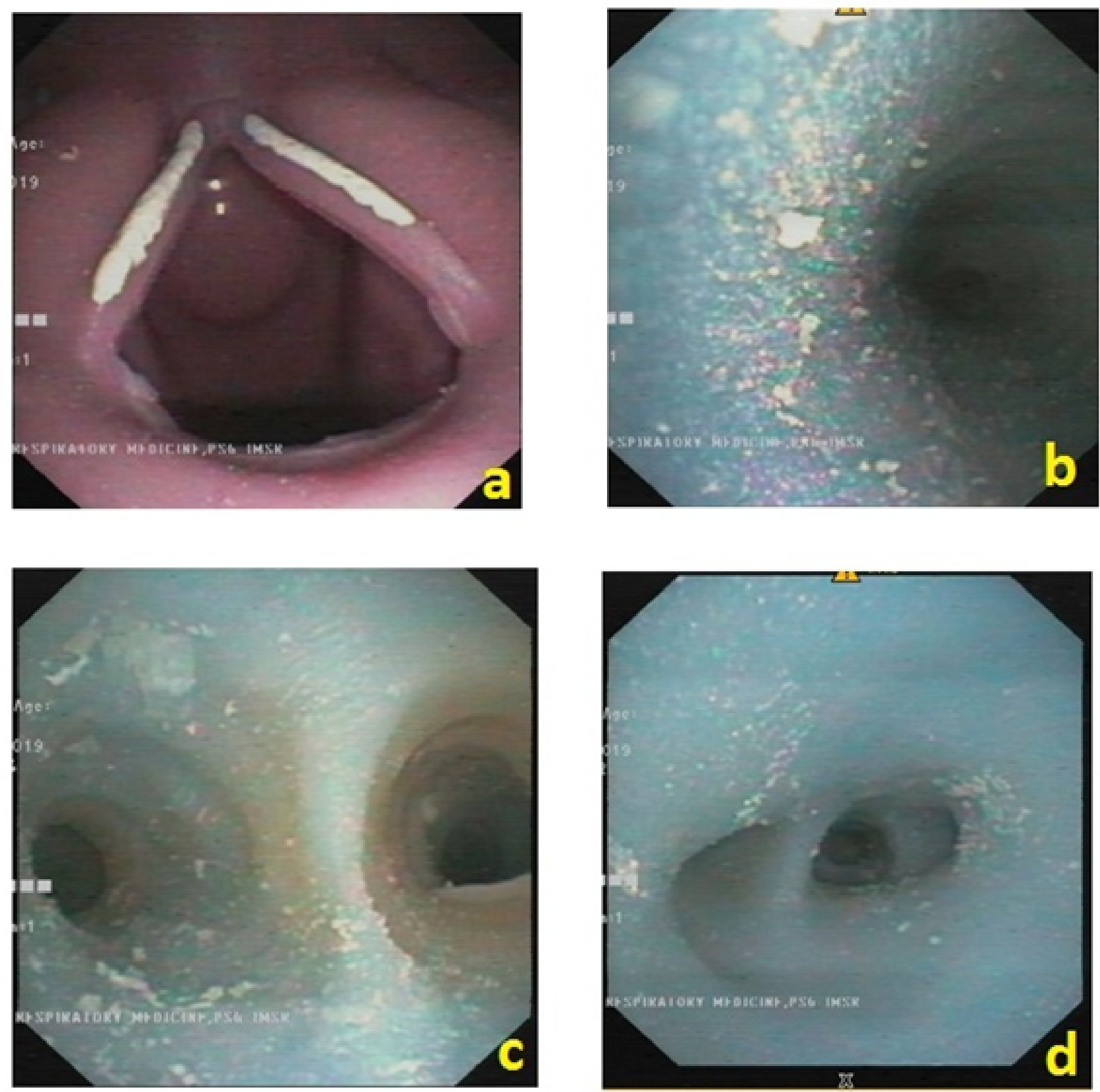

Figure 5: Images from the bronchoscope, a. Laryngeal inlet b. Trachea C. Carina, d. Right bronchus intermedius showing the lower lobe and middle lobe bronchi.

training needs to be imparted to the undergraduate medical students early in their career, relevant to the clinical posting they undergo. Apart from imbibing the required skills, an effective simulation program can also help the students to acquire cognitive aspects like guiding clinical decisions, gaining expertise in team dynamics, developing communication skills and leadership.

Since practising a surgical procedure or an invasive diagnostic technique for the first time on a real patient, even under supervision could be a dangerous proposition, and unethical. 
Skills training has to be done ideally using models and mannequins and to be successful, these models need to be made as life-like a manner as possible. The evolving science of clinical simulation is based on these prerequisites of effective learning practices. The efficacy of simulated learning depends upon how real the training system is, which is based on the quality of the mannequins, popularly termed as fidelity.

The role of the clinical simulation laboratory in equipping themselves to meet the challenges in providing the desirable learning environment and opportunities cannot be overemphasised. Teaching faculty at all levels of career also find it highly useful to enhance their skills and update techniques, using simulations.

Medical education is turning to be competency-based and it is in this context that the Medical Council of India also has laid down strict guidelines for imparting skills training during the undergraduate medical course, as part of competencybased medical education. Innovations have been developed which enable us to create customised patient-specific surgical models, custom-made prosthetics and surgical tools. Very recently evolved science of bioprinting enables us to grow live cells, using biocompatible materials. ${ }^{[1]}$ For bronchoscopy training, a regular 3D printed airway model has proved to be very useful and in fact, this technology has been accepted in a wide range of training scenarios, wherein custom-made 3D printed anatomical models have come to be used in clinical training scenarios. ${ }^{[12]}$

Simulation-based clinical training (SBCT) using 3D printed anatomical models provides the learner the opportunity of learning the skills in a safe and controlled environment, in as lifelike a manner as possible. ${ }^{[13,14]}$ SBCT depends upon using the correct simulation tools, which will mimic the real clinical scenario.

Even though there is a need to master very many clinical skills, a few of them are more important, by virtue of their direct relevance to more than one specialty of medicine. Training in bronchoscopy is one such skill. Apart from respiratory medicine, bronchoscopy is a technique that is widely used in the practice of many other specialties like anesthesiology, critical care and cardiothoracic surgery. Anatomical accuracy of the complex airway becomes crucial while producing a training system, which can simulate the respiratory conduits, required mastering this skill since the design and morphometry of the airway conduit is complex. ${ }^{[6]}$ While conventional anatomical models may be inadequate in producing the lifelike environment, which is required for simulated learning, 3D printed model alone would be able to provide the necessary training atmosphere. ${ }^{[15,16]}$

Such a model needs to reflect the exact branching pattern of the bronchial tree, bringing in the length, angulations and diameters of each generation of the airway. It also becomes necessary that the material with which the model is made need to have the required resilience, for the bronchoscope to travel through, retaining the toughness to withstand the force and thrust of the travel of the scope through the airway. This becomes crucial since the physician needs to steer through the airway with the bronchoscope till the specific bronchopulmonary segment is reached.

It is important that this complex anatomy of the tracheobronchial tree is reflected in the three-dimensional model and also the intraluminal appearance. The process of $3 \mathrm{D}$ printing starts from the base data of high-quality CT scan images. The software available with the CT scanner is able to provide a three-dimensional image of an organ, which provides smooth rendering, but these images lack clear tissue contrast. This software is not able to provide color-coding of different tissues and more importantly, it is not able to produce the physical model. Hence for an accurate replica of an anatomical model, 3D printing remains the most preferred choice. Among the very many digital formats compatible with $3 \mathrm{D}$ printing, STL remains the most dependable format and the conversion was made possible through 3D Doctor. Similarly, the choice of the software used in the process for computer-aided designing and final printing was dependant on the previous experience of the engineers involved in the project as well as ready accessibility of the software.

The training system was set up by attaching the 3D printed airway to a standard airway mannequin, using an adapter. Bronchoscope, lubricated with $2 \%$ lignocaine jelly and connected to a monitor was introduced to our training system and the various bronchopulmonary segments could be reached without difficulty. The system was checked by the faculty and was found to be acceptable for bronchoscopy training. Clinical scenarios could be presented to the residents, who went through the treatment protocol and clinical management of these scenarios. The training protocol, in principle, followed the general guidelines adopted in our SBCT programs. ${ }^{[11,14]}$ To our knowledge, 3D technology has not been explored well enough in applications pertaining to teaching and learning of medicine in India even though the technology is already being used in the therapeutic domain of medicine especially in the fields of dental sciences and orthopedics for manufacturing custom made prosthesis. The trials conducted so far have prompted the faculty to accept this bronchoscopy training model as a valuable resource to be included in our already existing list of programs of SBCT.

\section{Conclusions}

We have been able to create a 3D printed model of the tracheobronchial tree, starting from the base data of CT scan images of the thorax in a person with normal anatomy of the respiratory system. Using appropriate software, the CT scan 
DICOM images were converted into STL format and then 3D printed, using a combination of Veroclear and rubber. This model has proved to be of immense use in training the interns and the faculty in bronchoscopy, which requires the skill of accurately maneuvering through the airway and reaching all the bronchopulmonary segments, with detailed anatomy knowledge. This has enabled us to include bronchoscopy skills training in the already existing modules of our SBCT program.

\section{Acknowledgments}

Authors wish to acknowledge the help rendered by Dr. Rajesh, Professor and Mr. Arivazhagan, Research Assistant of Coimbatore Institute of Technology; Coimbatore for their help in printing this model.

\section{References}

1. ;. Available from: https://www.mciindia.org/char"203A relax2020/01 \char"203A|relaxSkill-Module_23.12.2019.pdf.

2. Hodgdon T, Danrad R, Patel MJ, Smith SE, Richardson ML, Ballard DH, et al. Logistics of Three-dimensional Printing. Acad Radiol. 2018;25(1):40-51. Available from: https://dx.doi. org/10.1016/j.acra.2017.08.003.

3. Ho BHK, Chen CJ, Tan GJS, Yeong WY, Tan HKJ, Lim AYH, et al. Multi-material three dimensional printed models for simulation of bronchoscopy. BMC Med Ed. 2019;19:236. Available from: https://doi.org/10.1186/s12909-019-1677-9.

4. Parotto M, Jiansen JQ, AboTaiban A, Ioukhova S, Agzamov A, Cooper R, et al. Evaluation of a low-cost, 3D-printed model for bronchoscopy training. Anestezjol Intens Ter. 2017;49(3):189-197. Available from: https://dx.doi.org/10. 5603/ait.a2017.0035.

5. Byrne T, Yong SA, Steinfort DP. Development and Assessment of a Low-Cost 3D-printed Airway Model for Bronchoscopy Simulation Training. J Bronchology Interv Pulmonol. 2016;23(3):251-254. Available from: https://dx.doi. org/10.1097/lbr.0000000000000257.

6. Ryan B, Yendamuri K, Yendamuri S. Anatomical considerations in bronchoscopy. J Thorac Dis. 2017;9(S10):S1123S1127. Available from: https://dx.doi.org/10.21037/jtd.2017. 08.116 .

7. Robinson RJ, Russo J, Doolittle RL. 3D Airway Reconstruction Using Visible Human Data Set and Human Casts with Comparison to Morphometric Data. Anat Rec (Hoboken). 2009;292(7):1028-1044. Available from: https://dx.doi.org/10. 1002/ar.20898.

8. Konge L, Arendrup H, von Buchwald C, Ringsted C. Using Performance in Multiple Simulated Scenarios to Assess Bron- choscopy Skills. Respiration. 2011;81(6):483-490. Available from: https://dx.doi.org/10.1159/000324452.

9. Stather DR, MacEachern P, Chee A, Dumoulin E, Tremblay A. Trainee Impact on Procedural Complications: An Analysis of 967 Consecutive Flexible Bronchoscopy Procedures in an Interventional Pulmonology Practice. Respiration. 2013;85(5):422428. Available from: https://dx.doi.org/10.1159/000346650.

10. Fielding DI, Maldonado F, Murgu S. Achieving competency in bronchoscopy: Challenges and opportunities. Respirology. 2014;19(4):472-482. Available from: https://dx.doi.org/10. 1111/resp. 12279.

11. Sigaux N, Pourchet L, Breton P, Brosset S, Louvrier A, Marquette CA. 3D Bioprinting:principles, fantasies and prospects. J Stomatol Oral Maxillofac Surg. 2019;120(2):128132. Available from: https://dx.doi.org/10.1016/j.jormas.2018. 12.014 .

12. Eley KA. Centralised 3D printing in the NHS: a radiological review. Clin Radiol. 2017;72:269-275. Available from: https: //doi.org/10.1016/j.crad.2016.12.013.

13. Anupamamurthy K, Ramaraju K, Balasubramanian N, Kumar PA. System based approach to improve competencies in respiratory emergencies amongst interns. Int J Innov Res Dev. 2015;4(11):213-217.

14. Nilsson PM, Naur TMH, Clementsen PF, Konge L, L. Simulation in bronchoscopy: current and future perspectives. Adv Med Educ Pract. 2017;8:755-760. Available from: https: //dx.doi.org/10.2147/AMEP.S139929.

15. Ernst A, Wahidi MM, Read CA, Buckley JD, Addrizzo-Harris DJ, Shah PL, et al. Adult bronchoscopy training: current state and suggestions for the future. Chest J. 2015;148:331-332. Available from: https://www.researchgate.net/deref/http\%3A/ /dx.doi.org/10.1378/chest.14-0678.

16. Kennedy CC, Maldonado F, Cook DA. Simulation based bronchoscopy training: systematic review and meta-analysis. Chest J. 2013;144(1):183-192. Available from: https://doi.org/ $10.1378 /$ chest.12-1786.

Copyright: (c) the author(s), 2020. It is an open-access article distributed under the terms of the Creative Commons Attribution License (CC BY 4.0), which permits authors to retain ownership of the copyright for their content, and allow anyone to download, reuse, reprint, modify, distribute and/or copy the content as long as the original authors and source are cited.

How to cite this article: Viyannan M, Kumar PA, Eswarswamy S, Murugesan G, Ramaraju K. 3D Printed Model of Airway for Clinical Simulation. Asian J. Med. Radiol. Res. 2020;8(2):50-56.

DOI: dx.doi.org/10.47009/ajmrr.2020.8.2.8

Source of Support: Nil, Conflict of Interest: None declared. 